\title{
HYDROLYSIS OF PLA-LIKE PLASMA POLYMER FILMS WITH VARYING DEGREE OF CROSSLINKING
}

\author{
Kolarova Raskova Z. ${ }^{a, *}$, Kousal J. ${ }^{b}$, Stloukal P. ${ }^{a}$, Krtous Z. ${ }^{b}$ \\ ${ }^{a}$ Centre of Polymer Systems, Tomas Bata University in Zlin, Trida Tomase Bati 5678, 76001 Zlin, Czech \\ Republic \\ ${ }^{b}$ Department of Macromolecular Physics, Faculty of Mathematics and Physics, Charles University in Prague, \\ VHolesovickach 2, 18200 Prague, Czech Republic \\ * zraskova@cps.utb.cz
}

\begin{abstract}
Poly-lactide acid (PLA) based biodegradable films are of interest for packaging materials or bioapplications. Plasma-assisted vacuum evaporation technique uses oligomers released during thermal decomposition of source polymer as precursors for plasma polymerization. Conventionally prepared PLA with $m w=10000 \mathrm{~g} / \mathrm{mol}$ was used as a source polymer. Films were prepared at various RF $(13.56 \mathrm{MHz})$ plasma powers $(0-20 \mathrm{~W})$ in order to vary the amount of crosslinking in the film.

Swelling and hydrolysis of films were monitored in real time using spectroscopic ellipsometry. The concentration profile of products of hydrolysis was measured by liquid-chromatography (LC-MS). FTIR, XPS and SEM analyses were used for monitoring of film composition and surface characterization. Molecular weights of source polymer and of the plasma polymer were determined by gel-permeation chromatography (GPC).

Possibility to prepare PLA-like plasma polymer films with controlled degradability by hydrolysis was demonstrated.
\end{abstract}

Keywords: polylactide, plasma polymer, biodegradability, hydrolysis.

\section{Introduction}

Biodegradable polymers are the topic of extensive research in recent years. Among the non-water-soluble polymers, the aliphatic polyester poly(lactic acid) (PLA) has been the most widely used in the biomedical field due to its unique biocompatibility, biodegradability and mechanical properties [1, 2]. Most often, PLA is synthetised by polycondensation of D- or Llactic acid or from ring opening polymerization of lactide, a cyclic dimer of lactic acid [3], however other synthetic methods have been also studied [4] 6 . Lowtemperature plasma based methods have proven to be very effective for fabrication of ultrathin, pinhole-free, polymeric and nanocomposite coatings [7 9].

Plasma polymerization from a thermally degraded solid polymer (plasma-assisted vacuum thermal evaporation) is most directly related to classical Physical Vapour Deposition (PVD) processes, but it utilizes plasma in the second step of the process. Long fragments of partially thermally decomposed bulk polymer fly through low-pressure plasma and they form a plasma polymer [9-13]. This method is able to produce thin films with relatively easily controllable cross-linking degree, as was demonstrated e.g. on PEG/PEO-like (polyethylene glycole/polyethylene oxide) films that otherwise needs bonding with crosslinking molecule if produced by wet chemistry processes. The thermal degradation occurs by random chain scission reactions, depolymerization, oxidative degradation, intramolecular and intermolecular transesteri- fications, hydrolysis, pyrolytic elimination and radical reactions 9 .

This deposition technique can adapt large variety of precursors ("source" polymers). When biodegradable precursors are used, an interesting question arises about the degradability of the resulting plasma polymer films. In this paper, first results from plasmaassisted vacuum thermal evaporation of PLA and of subsequent the study of PLA-like thin films with controlled hydrolysis kinetics are presented.

\section{Experimental}

\subsection{Deposition of plasma polymer}

The films were prepared in a setup described e.g. in [13. The gradually heated (from $25^{\circ} \mathrm{C}$ to $400^{\circ} \mathrm{C}$ ) crucible with a polymer precursor ("source" polymer) was placed $4 \mathrm{~cm}$ above the RF cathode with suppresed sputtering (using glass target, no Si was detected in the films). The substrates (single-side polished silicon wafers, gold-coated silicon, aluminium foil, glass slides) were placed $10 \mathrm{~cm}$ above the crucible. As a source polymer for the process, PLA prepared by polycondensation according to [14 was used. The chamber (pumped by rotary and diffusion pump) was filled with argon under the pressure of $4 \mathrm{~Pa}$ and the flow rate of $8 \mathrm{sccm}$. An rf generator (Dressler Cesar, $13.56 \mathrm{MHz}$ ) with an automatic matching unit (MFJ-962D) was used to deliver power to the electrode, see Figure 11 Depositions were performed at rf powers into the plasma electrode of $0,2,5$ and $20 \mathrm{~W}$. 


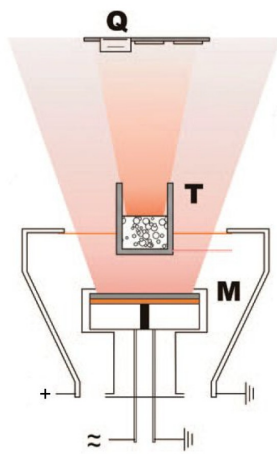

Figure 1. Scheme of plasma assisted vacuum thermal evaporation. $T$ - heating cell with source polymer, $M$ plasma source, Q - substrates. (adapted from [13])

The deposition rate was monitored by quartz-crystal microbalance sensor.

\subsection{Determination of composition of films}

The weight average molecular weight $(\mathrm{Mw})$ and $\mathrm{Mw}$ distribution of PLA prior to and after deposition were analysed by gel permeation chromatography (GPC) on an HT-GPC 220 system (Agilent). Samples on aluminiom foil were dissolved in tetrahydrofuran (THF) $\left(2 \mathrm{mg} . \mathrm{ml}^{-1}\right)$ overnight. Separation and detection took place on a series of mixed columns $(1 \times B, 1 \times D, 1 \times E)$ $(300 \times 7.8 \mathrm{~mm}$, Polymer Laboratories $)$. Analyses were carried out at $40^{\circ} \mathrm{C}$ in THF, $1.0 \mathrm{ml}$.min ${ }^{-1}$ flow rate and a loading volume of $100 \mu \mathrm{L}$. The elemental composition of the films in dry state was analyzed by XPS (Phoibos 100, Specs). FTIR-ATR (Nicolet iS5) were recorded for samples on gold-precoated substrates before and after hydratation/hydrolysis in climatic box BMT Climacell with $84 \%$ relative humidity at $24^{\circ} \mathrm{C}$ for 1 month.

\subsection{Characterization of hydrolysis}

Ellipsometric measurements (Woollam M-2000DI) were first performed on the films on silicon to find the thickness of the films in dry state. Subsequently, the swelling and hydrolysis of the films in water was monitored in-situ for 180 minutes. The hydrolysis experiments were carried out on samples on the glass slides at $37^{\circ} \mathrm{C}$ in $15 \mathrm{ml}$ ammonium bicarbonate buffer $(0.01$ mol $\mathrm{L}^{-1}$, pH 7) amended with a microbial growthinhibiting substance (sodium azide, $0.2 \% \mathrm{w} / \mathrm{w}$ ). 0.5 $\mathrm{mL}$ aliquots of the medium were taken at regular intervals, centrifuged and were analysed for lactic acid by LC-MS (Agilent 6530 Accurate Mass Q-TOF LC/MS with electrospray ionization source) coupled to an HPLC unit (Agilent 1260 Infinity). Chromatographic analysis was conducted on large pore core-shell column for reversed phase separation Agilent Aeris Widepore XB-X8.

\section{Results}

The thickness of the film as-deposited was 100-300 nm. The FTIR spetra show that the basic structural units

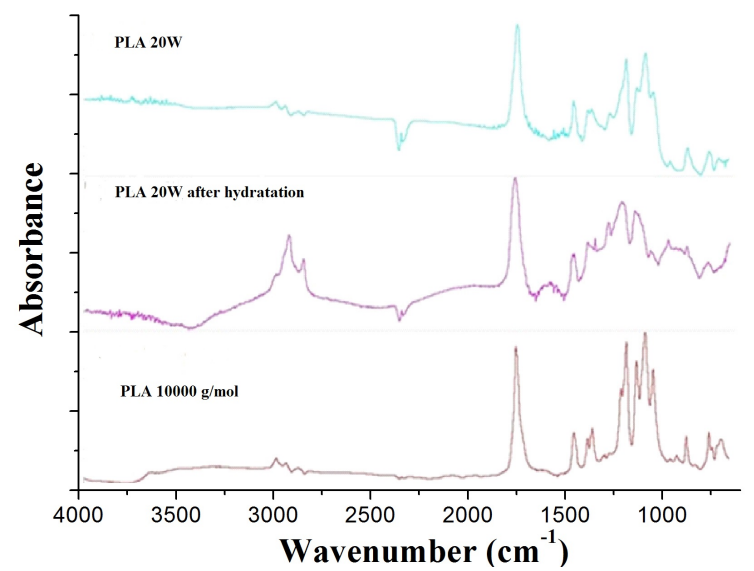

Figure 2. FTIR-ATR spectra (top to bottom): thin film prepared at the power $20 \mathrm{~W}$ as deposited, the same thin film after hydratation in climatic box, PLA precursor ("source" polymer).

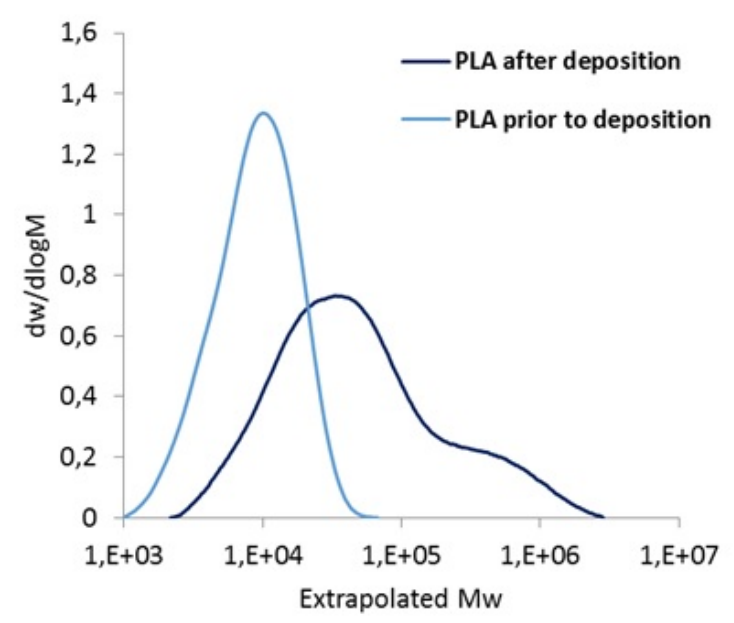

Figure 3. Molecular weight distribution for PLA precursor and PLA-like polymer (without plasma).

of PLA polymer were well preserved in the films as well. Even for films prepared at the highest power $(20 \mathrm{~W})$ the infrared fingerprint region below $1000 \mathrm{~cm}^{-1}$ shows rather minor qualitative differences from the source polymer (Figure 2). Presence of ester bonds $\mathrm{C}-\mathrm{O}-\mathrm{C}$ is shown in band at $1040 \mathrm{~cm}^{-1}$ and $\mathrm{v}(\mathrm{C}=\mathrm{O})$ spectral band was observed at $1748 \mathrm{~cm}^{-1}$. Bands near $3000 \mathrm{~cm}^{-1}$ can be assigned to the $\mathrm{CH}_{x}$ stretching vibrations. The elemental composition of the films using XPS shows that for low plasma powers (up to $2 \mathrm{~W}) \mathrm{C} / \mathrm{O}$ ratio is close to 1.5 that matches the $\mathrm{C} / \mathrm{O}$ ratio for PLA (1.5). For plasma powers from 5 to $20 \mathrm{~W}$ the $\mathrm{C} / \mathrm{O}$ decreases to about 1.3 due to higher amount of $\mathrm{C}=\mathrm{O}$ groups.

Results obtained from GPC (Figure 3) on the films prepared from thermal evaporation without plasma reveal marked differences in molecular weight and also in its distribution in comparison to original PLA polymer. PLA that was used as a precursor show relatively narrow distribution of molecule chain lengths. The plasma polymer exhibits significantly higher poly- 


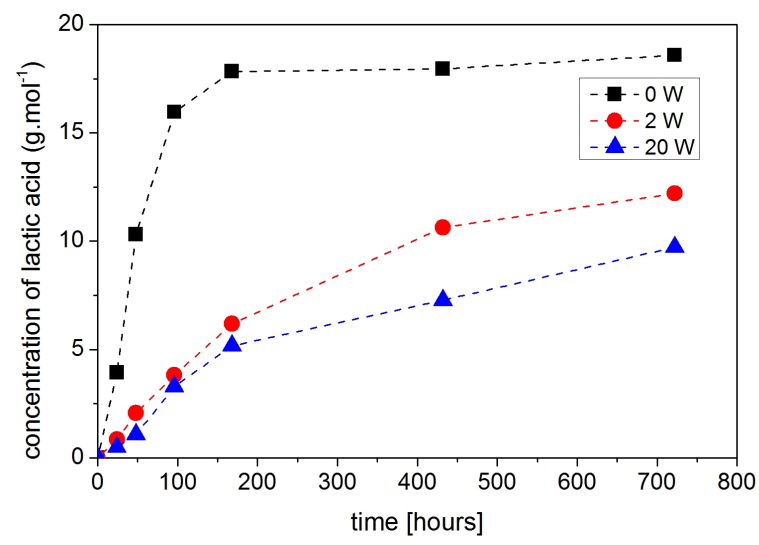

Figure 4. Lactic acid release from hydrolyzed PLA plasma polymer films determined by LC-MS for films prepared at RF powers $0-20 \mathrm{~W}$.

dispersity and molecular weight. Molecular weight of plasma polymer was $50 \mathrm{~kg} / \mathrm{mol}$, while molecular weight of precursor was $10 \mathrm{~kg} / \mathrm{mol}$. This can be attributed to recombination and crosslinking of radicals from the chains released during the thermal degradation of the source polymer.

Monitoring of hydrolysis of plasma polymer in terms of lactic acid release is shown in Figure 4 The lactic acid release was higher during the first $24-50$ hours, noticeably faster for the films deposited without plasma discharge. After about 200 hours of immersion, the lactid acid release rate decreased gradually. A diffusion behavior according to the classical Fickian diffusion phenomenon has been reported previously in other PLA products [5]. The lactic acid release gets slower and less pronounced with the increase of the discharge power during thin film deposition as can be expected for more crosslinked films. The in-situ monitoring of thin films degradation in water using spectroscopic ellipsometry (Figure 5) revealed a nontrivial kinetics of initial stages of the process of film hydratation. The time dependence of thickness of the film in water shows competetive processes of swelling of the plasma polymer and its hydrolysis. With increasing RF power during deposition, the moment of attaining maximum thickness (swelling) is time-delayed and PLA dissolving is slower. Only in case of the film prepared at $0 \mathrm{~W}$ the 3 hours were enough to reach the equilibrium state. Additionally, films that were hydrated in climatic box, show changes the in the infrared spectra in mainly at $1550 \mathrm{~cm}^{-1} \mathrm{C}=\mathrm{O}$ bands and at $\mathrm{OH}$ groups at $3000 \mathrm{~cm}^{-1}$ as expected for accelerated oxidation and hydrolysis (Figure 22. General broadening of the peaks in the fingerprint region is another marker of polymer chain scission and fragmentation.

Both chemical and ellipsometric characterization of the swelling clearly show that the degradability can be controlled via plasma deposition conditions.

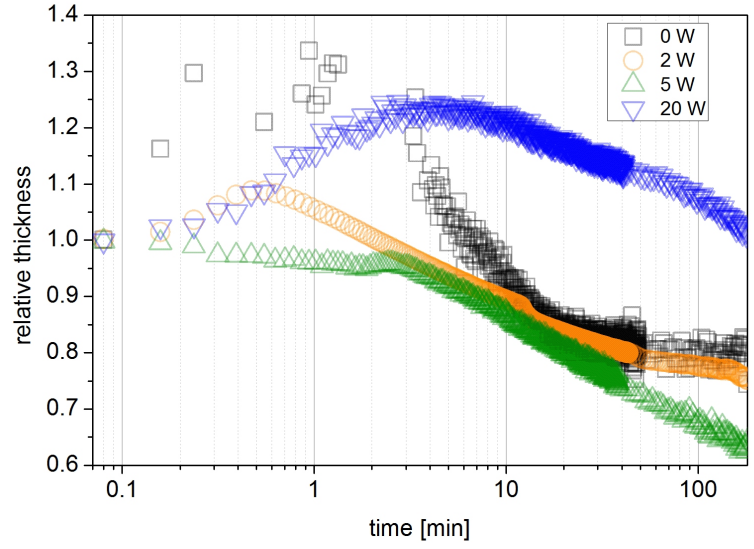

Figure 5. Kinetics of swelling and hydrolysis of the films in water observed in-situ by ellipsometry for films prepared at $R F$ powers $0-20 \mathrm{~W}$.

\section{Conclusions}

Novel PLA-like polymer were succesfully prepared using plasma-assisted vacuum thermal evaporation. The chemical structure of the films very well corresponds to the original PLA source polymer. It was demonstrated that the hydrolysis kinetics and water solubility of the films can be controlled by deposition conditions, namely the discharge power. This technique is promising for the preparation of films with controlled permeability, biodegradabilty or drug release for medical applications.

\section{Acknowledgements}

Authors gratefully acknowledge to the Ministry of Agriculture of the Czech Republic (grant No. QJ1310254) and the Ministry of Education, Youth and Sports of the Czech Republic, Program NPU I (LO1504) and the Student Grant of the Faculty of Mathematics and Physics of Charles University in Prague "Regulation of stability of biodegradable polymers".

\section{References}

[1] C.-S.Ha, J.A.Gardella Jr.: Surface Chemistry of Biodegradable Polymers for Drug Delivery Systems, Chem. Rev., (105), 4205-4232, 2005

[2] Zhou H. ,. Lawrence J.G. , Bhaduri S.B., Fabrication aspects of PLA-CaP/PLGA-CaP composites for orthopedic applications: A review, Acta Biomaterialia (8), 1999-2016, 2012

[3] Luo Y.B. , Wang X.L. , Zhong Wang Y. , Effect of $\mathrm{TiO} 2$ nanoparticles on thelong-term hydrolytic degradation behavior of PLA, Polymer Degradation and Stability (97), 721-728, 2012

[4] H.Tian, Z.Tang, X.Zhuang, X.Chen, X.Jing: Biodegradable sythetic polymers: Preparation, functionalization and biomedical application, Progress in Polymer Science, (37) 237-280, 2012

[5] Oliveira M., Santos E., Araujo A., Fechine G.J.M., Machado A.V., Botelho G., The role of shear and stabilizer on PLA degradation, Polymer Testing (51), 109-116, 2016 
[6] Witting M., Obst K., Friess W., Hedtrich S., Recent advances in topical delivery of proteins and peptides mediated by soft matter nanocarriers, Biotechnology Advances (33), 1355-1369, 2015

[7] Friedrich, J. Mechanisms of Plasma Polymerization-Reviewed from a Chemical Point of View. Plasma Processes Polym. 8 (9), 783-802, 2011

[8] Fahmy, A., Mix, R.; Schoonhals, A.; Friedrich, J. Structure of Plasma-Deposited Copolymer Films Prepared from Acrylic Acid and Styrene: Part II Variation of the Comonomer Ratio. Plasma Processes Polym. 10 (9), 750-760, 2013

[9] Choukourov A., Gordeev I., Ponti Chiara Uboldi J., Melnichuk I., Vaidulych M., Kousal J., Nikitin D., HanykovaİĄ L., KrakovskyİĄ I., SlavÃnnnska D., Biederman H, Microphase-Separated PE/PEO Thin Films Prepared by Plasma-Assisted Vapor Phase Deposition, ACS Appl. Mater. Interfaces, 8

(12),8201-8212, 2016

[10] Koster G., Rijnders G., In Situ Characterization of Thin Film Growth, Cambridge, Woodhead Publishing, UK, 296 p., 2011

[11] Siow K., Kumar S., Griesser H.J., Low-pressure plasma methods for generating non-reactive hydrophilic and hydrogel-like bio-interface coatings, Plasma Process Polym.,12(1), 8-24, 2014

[12] Choukourov A., Gordeev I., Arzhakov D., Artemenko A., Kousal J., Kylian O., Slavinska D., Biederman H., Does CrossLink Density of PEOLike Plasma Polymers Influence their Resistance to Adsorption of Fibrinogen?, Plasma Processes and Polymers, vol. 9 (1),48-58, 2011

[13] Choukourov A., Grinevich A., Polonskyi O., Hanus J., Kousal J., Slavinska D., Biederman H., Vacuum Thermal Degradation of Poly(ethylene oxide), J. Phys. Chem. B, 113 (10), 2984-2989, 2009

[14] Kucharczyk P., Dvorak Z., Hnatkova E, Sedlarik V., Novel aspects of the degradation process of PLA based bulky samples under conditions of high partial pressure of water vapour Polymer Degradation and Stability 98(1), 150-157, 2013 